\title{
Evidence of a geomagnetic effect on extensive air showers detected with the ARGO-YBJ experiment
}

B. Bartoli, ${ }^{1,2}$ P. Bernardini, ${ }^{3,4, *}$ X. J. Bi, ${ }^{5}$ I. Bolognino, ${ }^{6,7}$ P. Branchini,${ }^{8}$ A. Budano, ${ }^{8}$ A. K. Calabrese Melcarne, ${ }^{3,4}$ P. Camarri, ${ }^{9,10}$ Z. Cao,${ }^{5}$ R. Cardarelli, ${ }^{10}$ S. Catalanotti, ${ }^{1,2}$ S. Z. Chen,${ }^{5}$ T. L. Chen,${ }^{11}$ Y. Chen, ${ }^{5}$ P. Creti, ${ }^{4}$ S. W. Cui, ${ }^{12}$ B.Z. Dai, ${ }^{13}$ A. D'Amone, ${ }^{3,4}$ Danzengluobu, ${ }^{11}$ I. De Mitri, ${ }^{3,4}$ B. D'Ettorre Piazzoli, ${ }^{1,2}$ T. Di Girolamo, ${ }^{1,2}$ X. H. Ding ${ }^{11}$ G. Di Sciascio, ${ }^{10}$ C. F. Feng, ${ }^{14}$ Zhaoyang Feng, ${ }^{5}$ Zhenyong Feng, ${ }^{15}$ Q. B. Gou, ${ }^{5}$ Y. Q. Guo, ${ }^{5}$ H. H. He, ${ }^{5}$ Haibing Hu, ${ }^{11}$ Hongbo Hu, ${ }^{5}$ Q. Huang, ${ }^{15}$ M. Iacovacci, ${ }^{1,2}$ R. Iuppa,${ }^{9,1}$ H. Y. Jia, ${ }^{15}$ Labaciren, ${ }^{11}$ H. J. Li, ${ }^{11}$ J. Y. Li, ${ }^{14}$ X. X. Li, ${ }^{5}$ G. Liguori, ${ }^{6,7}$ C. Liu, ${ }^{5}$ C. Q. Liu, ${ }^{13}$ J. Liu, ${ }^{13}$ M. Y. Liu, ${ }^{11}$ H. Lu,${ }^{5}$ X. H. Ma ${ }^{5}$ G. Mancarella, ${ }^{3,4}$ S. M. Mari, ${ }^{8,16}$ G. Marsella, ${ }^{3,4}$ D. Martello, ${ }^{3,4}$ S. Mastroianni, ${ }^{2}$ P. Montini, ${ }^{8,16}$ C. C. Ning, ${ }^{11}$ M. Panareo,${ }^{3,4}$ B. Panico, ${ }^{9,10}$ L. Perrone, ${ }^{3,4}$ P. Pistilli, ${ }^{8,16}$ F. Ruggieri, ${ }^{8}$ P. Salvini, ${ }^{7}$ R. Santonico, ${ }^{9,10}$ S. N. Sbano, ${ }^{3,4}$ P. R. Shen, ${ }^{5}$ X. D. Sheng, ${ }^{5}$ F. Shi, ${ }^{5}$ A. Surdo, ${ }^{4}$ Y. H. Tan, ${ }^{5}$ P. Vallania, ${ }^{17,18}$ S. Vernetto, ${ }^{17,18}$ C. Vigorito, ${ }^{18,19}$ B. Wang, ${ }^{5}$ H. Wang,,${ }^{5}$ C. Y. Wu, ${ }^{5}$ H. R. Wu, B. Xu, ${ }^{15}$ L. Xue, ${ }^{14}$ Y. X. Yan, ${ }^{13}$ Q. Y. Yang, ${ }^{13}$ X. C. Yang, ${ }_{13}^{13}$ Z. G. Yao, ${ }^{5}$ A. F. Yuan, ${ }^{11}$ M. Zha, ${ }^{5}$ H. M. Zhang, ${ }^{5}$ Jilong Zhang, ${ }_{15}$ Jianli Zhang, ${ }^{5}$ L. Zhang, ${ }^{13}$ P. Zhang, ${ }^{13}$ X. Y. Zhang, ${ }^{14}$ Y. Zhang, ${ }^{5}$ Zhaxiciren, ${ }^{11}$ Zhaxisangzhu, ${ }^{11}$ X. X. Zhou, ${ }^{15}$ F. R. Zhu, ${ }^{15}$ Q. Q. Zhu, ${ }^{5}$ and G. Zizzi ${ }^{20}$

(ARGO-YBJ Collaboration)

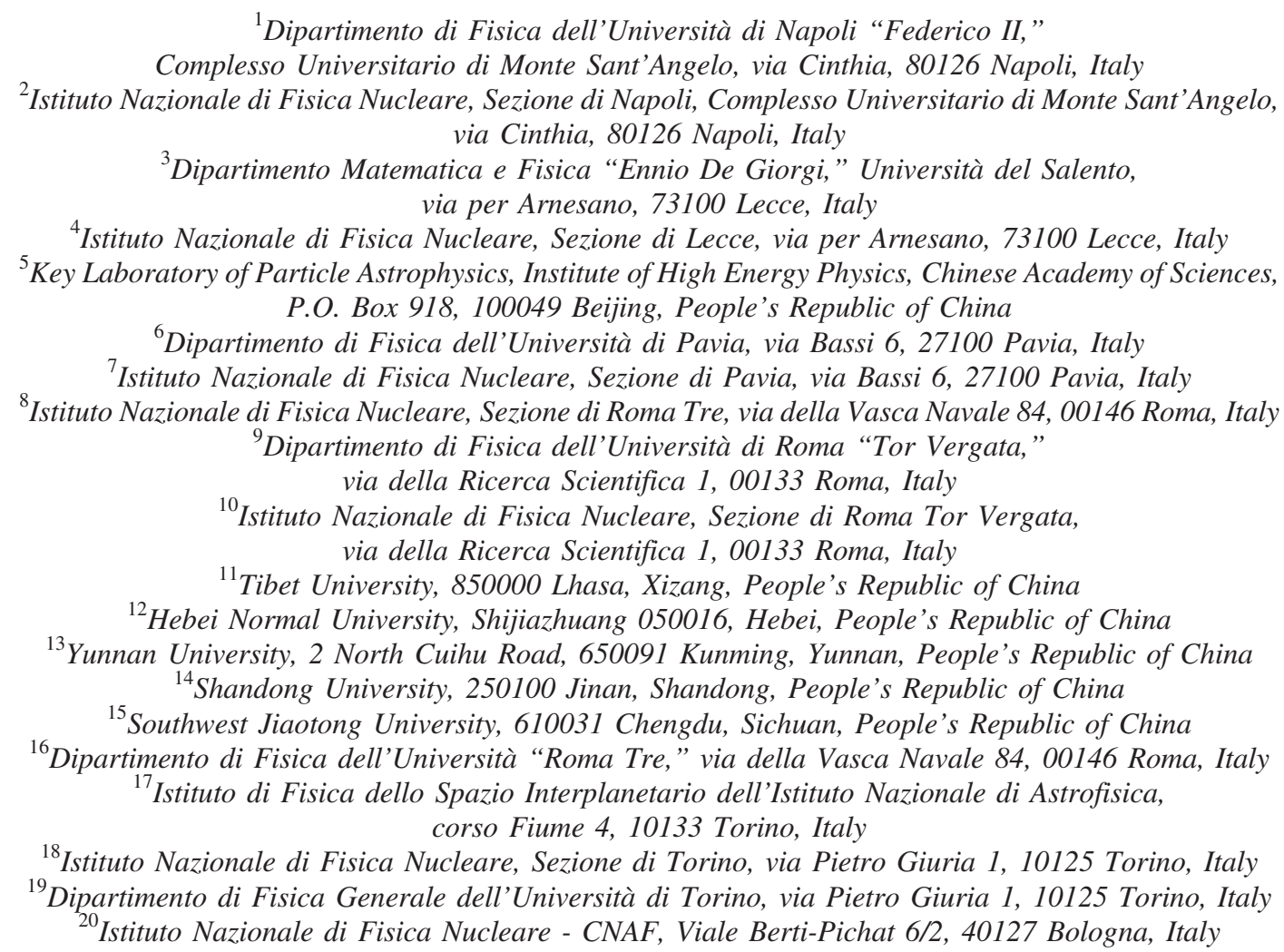

(Received 31 July 2013; published 28 March 2014)

The geomagnetic field causes not only the east-west effect on primary cosmic rays but also affects the trajectories of the secondary charged particles in the shower, causing their lateral distribution to be stretched. Thus, both the density of the secondaries near the shower axis and the trigger efficiency of detector arrays decrease. The effect depends on the direction of the showers, thus, introducing a modulation in the measured azimuthal distribution. The azimuthal distribution of the events collected by the ARGO-YBJ detector is deeply investigated for different zenith angles in light of this effect.

DOI: 10.1103/PhysRevD.89.052005

PACS numbers: 91.25.-r, 95.85.Ry, 96.50.sd

*Corresponding author. paolo.bernardini@le.infn.it 


\section{INTRODUCTION}

The path of charged primary cosmic rays (CRs) is deflected by magnetic fields. The galactic magnetic field randomizes the $\mathrm{CR}$ arrival directions. The geomagnetic field (GeoMF) prevents low-rigidity CRs from reaching the terrestrial atmosphere and causes the east-west asymmetry (the CR flux from the east is lower than that from the west) [1]. The GeoMF acts also on the charged particles of the extensive air showers (EAS) during their travel in the atmosphere. Cocconi [2] suggested that the lateral displacement induced by the Earth's magnetic field is not negligible with respect to the Coulomb scattering when the shower is young. According to Cocconi's model, the effect could increase for high-altitude measurements. Moreover, if the trigger efficiency of an array is sensitive to the shower lateral extension, the GeoMF can change the acquisition rate as a function of the zenith and azimuth angles. An azimuthal modulation was observed at the Yakutsk array for EAS with energies above $50 \mathrm{PeV}[3]$ and at the ALBORZ Observatory for energies above $100 \mathrm{TeV}$ [4]. The GeoMF effect has been observed in radio experiments [5] and studied to improve the EAS simulation [6], to correct the pointing of Čerenkov telescopes [7], and to discriminate the primary mass [8]. The importance of the GeoMF has been pointed out also in studies on CR large-scale anisotropy [9].

The GeoMF effect in the ARGO-YBJ data has been preliminarily estimated [10] and studied [11]. Here those studies are updated using a very large data sample, and for the first time, the GeoMF lateral stretching is observed at small distances from the shower core.

In Sec. II, the detector features are briefly presented. A simple model of the GeoMF action is sketched in Sec. III, and the results are confirmed by the simulation of CR beams (Sec. IV) assuming different values of the magnetic field. In Sec. V, the modulation of the azimuthal distribution is foreseen as an effect of the GeoMF action. The main data analysis is described in Sec. VI looking for hints of the GeoMF effect in a sample of showers with cores inside the detector. In Sec. VII, showers with external cores are also analyzed, and a check of the pointing accuracy is suggested in Sec. VIII. The conclusions are given in Sec. IX.

\section{THE ARGO-YBJ DETECTOR}

ARGO-YBJ [12] is an array located at the Yangbajing Cosmic Ray Observatory (Tibet, People's Republic of China), $4300 \mathrm{~m}$ above sea level $\left(90^{\circ} 31^{\prime} 50^{\prime \prime} \mathrm{E}, 30^{\circ} 06^{\prime} 38^{\prime \prime} \mathrm{N}\right)$. It is mainly devoted to $\gamma$-ray astronomy [13] and cosmic ray physics [14]. The detector consists of a $74 \times 78 \mathrm{~m}^{2}$ carpet made of a single layer of resistive plate chambers (RPCs) with $\sim 92 \%$ of active area surrounded by a partially instrumented $(\sim 20 \%)$ area up to $100 \times 110 \mathrm{~m}^{2}$. The apparatus has a modular structure, the basic data acquisition element being a cluster $\left(5.7 \times 7.6 \mathrm{~m}^{2}\right)$ made up of $12 \mathrm{RPCs}\left(2.8 \times 1.25 \mathrm{~m}^{2}\right.$ each). The full experiment is made up of 153 clusters for a total active surface of $\sim 6600 \mathrm{~m}^{2}$. The RPCs are operated in streamer mode with a gas mixture (Ar 15\%, isobutane $10 \%$, and tetrafluoroethane $75 \%$ ) suitable for high-altitude operation. Each RPC is read by 80 external strips of $6.75 \times 61.8 \mathrm{~cm}^{2}$ (the spatial pixels) that are logically organized in ten independent pads of $55.6 \times 61.8 \mathrm{~cm}^{2}$ each, which are individually acquired and represent the time pixels [15]. In addition, in order to extend the dynamical range up to $\mathrm{PeV}$ energies, each RPC is equipped with two large pads $\left(1.39 \times 1.23 \mathrm{~m}^{2}\right.$ each $)$ to collect the total charge of the particles hitting the detector [16].

ARGO-YBJ operates in two independent acquisition modes: shower and scaler mode [17]. Here the data collected by the digital readout in shower mode have been used. In this mode, an electronic logic has been implemented to build an inclusive trigger based on the time correlation between the pad signals, depending on their relative distances. The showers firing at least $N_{\text {trig }}$ pads in the central carpet in a time window of 420 ns generate a trigger. The time of each fired pad within $2 \mu$ s around the trigger time and its location are recorded and used to reconstruct the position of the shower core and the direction of the primary particle.

ARGO-YBJ started taking data with its complete layout in November 2007 with the trigger condition $N_{\text {trig }}=20$. The detector was operated until February 2013 with a duty cycle higher than $86 \%$, a trigger rate of $\sim 3.5 \mathrm{kHz}$ and a dead time of $4 \%$.

Typically, the EAS collected digitally in the shower mode have an energy in the range of 1-200 TeV, well beyond the rigidity cutoff at the YBJ site. Therefore, the effect of the GeoMF on the primary trajectory is negligible.

\section{A. Reference frame}

In the ARGO-YBJ reference system, the azimuth angle $(\phi)$ of the EAS is defined with respect to the detector axes in the counterclockwise direction $\left(\phi=0^{\circ}\right.$ for showers aligned with the $x$ axis and moving towards the positive direction). Thus, the azimuth angle of showers going towards the geographical north is $\phi_{N}=71.96^{\circ} \pm 0.02^{\circ}$. The quoted error is due to the measurement of the orientation of the detector axes with respect to the geographical reference system.

\section{B. Magnetic features of the site}

According to the International Geomagnetic Reference Field model available on the NOAA website [18], the geomagnetic field at YBJ in October 2010 had the following features (module, zenith, and azimuth, respectively) in the ARGO-YBJ reference system:

$$
B=49.7 \mu \mathrm{T}, \quad \theta_{B}=46.4^{\circ}, \quad \phi_{B}=71.89^{\circ} .
$$

The Lorentz force on the EAS charged particles depends on the quantity $B \sin \xi$, where $\xi$ is the angle between $\vec{B}$ and 


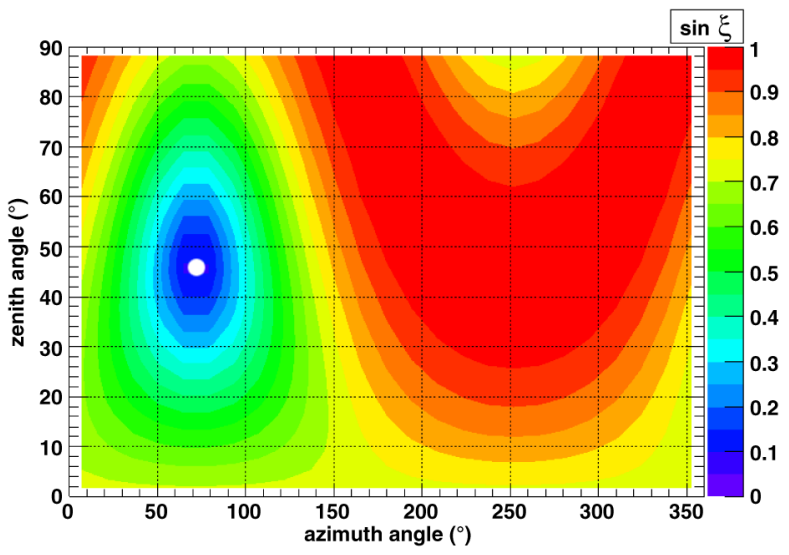

FIG. 1 (color online). Value of $\sin \xi$ versus the zenith and azimuth angles of the arrival direction of a charged particle in the ARGO-YBJ reference frame ( $\xi$ is the angle in between the magnetic field and particle direction). The white dot corresponds to particles moving parallel to $\vec{B}$.

the particle momentum $\vec{p}$. The value of $\sin \xi$ versus the zenith $(\theta)$ and azimuth of a particle at the YBJ site is displayed in Fig. 1. Then the magnetic effect is expected almost null in a small angular region $\left(\theta \sim 46^{\circ}, \phi \sim 72^{\circ}\right)$ and maximum in a larger belt with center in $\theta \sim 44^{\circ}$ and $\phi \sim 252^{\circ}$.

Both the intensity and the direction of the GeoMF vary in time and depend on the altitude, but the variations are very small [18]. The variations of $B, \theta_{B}$, and $\phi_{B}$ in a year are, respectively, $+40.3 \mathrm{nT},+0.1^{\circ},-0.01^{\circ}$. From 4.3 up to $30 \mathrm{~km}$ above sea level, the variation of the quantity $B \sin \xi$ is less than $2 \%$. Then the magnetic field can be assumed uniform and constant in this analysis of eight days of data.

\section{SIMPLE MODEL OF THE GeoMF EFFECT}

The trajectory of the EAS charged particles is deflected by the GeoMF in the plane perpendicular to $\vec{B}$ (hereafter named the bending plane). Along the direction of the GeoMF, the force is null and the velocity does not change. In the bending plane, the trajectory is circular with a radius $r$ defined according to

$$
r=\frac{p_{\perp}}{q B},
$$

where $p_{\perp}=p \sin \xi$ is the particle momentum in the bending plane and $q$ its electric charge. The corresponding angular velocity is

$$
\omega=\frac{q v_{\perp} B}{p_{\perp}}=\frac{q B}{\gamma m} .
$$

The particles are shifted along the west-east (WE) axis. Neglecting another possible displacement on the southnorth $(\mathrm{SN})$ axis, the time of flight $(\tau)$ of the particles is simply $\tau=L / \beta c$, where $L$ is the path length. The value $(d)$ of the shift on the shower front (Fig. 2) is expected to be

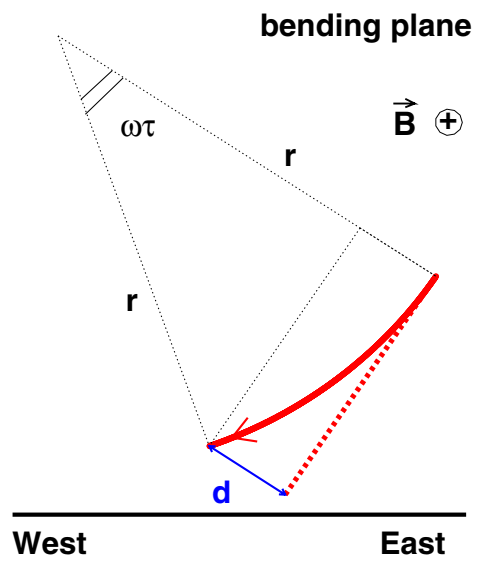

FIG. 2 (color online). The path of a secondary charged particle (bold red arrow) is modified by the GeoMF in the bending plane. The bold dotted red line is the particle path in absence of the GeoMF (the shower front is perpendicular to this line). The length $d$ is the lateral shift on the shower front due to the magnetic effect. The black dotted lines are drawn in order to explain the formulas in Sec. III.

$$
d=r[1-\cos (\omega \tau)] .
$$

Assuming small angular deviations $(\omega \tau \rightarrow 0)$ and relativistic particles $(\beta \simeq 1)$, it results

$$
d=\frac{p_{\perp}}{2 q B}(\omega \tau)^{2}=\frac{q L^{2}}{2 p} B \sin \xi .
$$

This shift of the charged particle path in the bending plane is the main effect of the GeoMF action. As a consequence of this WE shift, there is a time-of-flight small variation $(\Delta \tau)$, and taking into account the velocity component $\left(v_{\|}\right)$ parallel to $\vec{B}$, a small shift $\left(v_{\|} \Delta \tau\right)$ is expected also on the $\mathrm{SN}$ axis.

Then, Eq. (5) does not fully describe the GeoMF effect. Moreover, the model should take into account that each particle in the shower has different values of $p, \theta, \phi$, and $L$. In short, a Monte Carlo simulation is necessary in order to foresee the geomagnetic effect as a function of the shower axis direction. Anyway, Eq. (5) indicates an enlargement of the shower footprint. This implies a decrease of the particle density near the shower core, which is then balanced by an increase at larger distances, as pointed out in Ref. [3]. As a consequence. a very small, direction-dependent reduction in the ARGO-YBJ trigger efficiency can be envisaged for showers with the core lying inside the carpet. This and other features of the GeoMF effect have been simulated, and the results are presented in the next section.

\section{SIMULATION OF PRIMARY PROTON BEAMS}

Systematic uncertainties due to assumptions adopted in a full simulation approach (primary spectrum, chemical composition, interaction model, and so on) do not allow 
the study of tiny effects at the level of a few percent or less. Therefore, we preferred to simulate beams of primary protons in order to get the functional shape of the GeoMF and detector effects, whereas the huge statistics of the real data sample allows a model-independent estimate of the amplitudes of these effects.

The CORSIKA code [19] has been used to reproduce the shower development. The hadronic interactions above $80 \mathrm{GeV}$ are reproduced by the SIBYLL models [20], and the low energy hadronic interactions are described by the FLUKA package [21]. A GEANT3-based code [22] is used to simulate the detector response. The primary trajectory has been projected on a $10 \times 10 \mathrm{~m}^{2}$ ground area at the center of the carpet. The simulated data are studied with the same analysis chain applied to the real data. Hereafter, the angular coordinates $(\theta, \phi)$ define the direction of the shower axis, not that of a single particle.

\section{A. Detector acceptance}

At first, the effect of the detector acceptance was studied by simulating the showers in absence of the GeoMF. Proton beams were simulated with the same values of the primary energy (1 TeV), zenith angle $\left(27^{\circ}\right)$, interaction height $(19 \mathrm{~km})$, and five different values of the azimuth angle $\left(\phi=0^{\circ}, 45^{\circ}, 90^{\circ}, 135^{\circ}, 180^{\circ}\right)$. The primary energy and zenith angle were chosen because they were typical values of the data sample collected by the ARGO-YBJ experiment. As a result of the simulation, the azimuthal distribution of the rate $(\lambda)$ is modulated and can be fitted by the function

$$
\lambda=\lambda_{0}\left\{1+g_{2 A} \cos \left[2\left(\phi-\phi_{2 A}\right)\right]\right\},
$$

where the indexes 2 and $A$ refer to the second harmonic and the acceptance effect, respectively, and $\lambda_{0}$ is the mean rate. The fit parameters result in $g_{2 A}=(0.2094 \pm 0.0046) \%$ and $\phi_{2 A}=88^{\circ} \pm 7^{\circ}$.

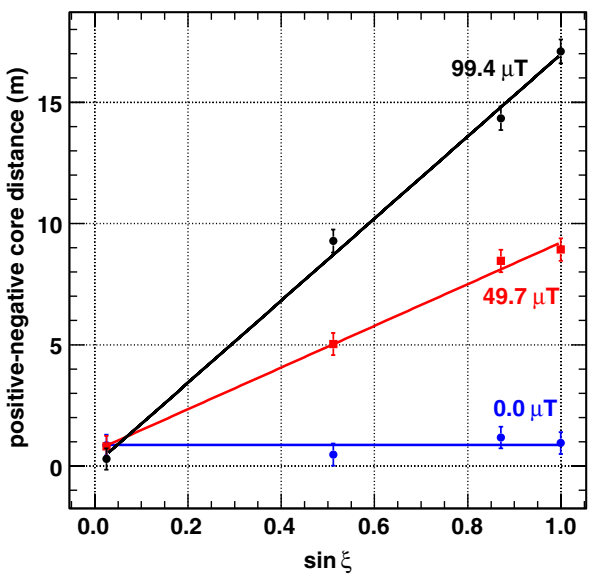

TABLE I. Angular features of the CR beams simulated to study the geomagnetic effect. The $\sin \xi$ range is fully covered.

\begin{tabular}{lcc}
\hline \hline$\theta\left(^{\circ}\right)$ & $\phi\left(^{\circ}\right)$ & $\sin \xi$ \\
\hline 45 & 71.5 & 0.025 \\
45 & 115.5 & 0.513 \\
45 & 161.5 & 0.871 \\
45 & 251.5 & 1.000 \\
\hline \hline
\end{tabular}

A simple geometrical simulation was performed in order to understand the origin of this azimuthal modulation with the maximum at $\sim 90^{\circ}$ and periodicity $180^{\circ}$. Modifying the detector geometry, we verified that it is due to the detector $x$ - $y$ asymmetry and increases with the zenith angle $\left(g_{2 A} \propto \operatorname{tg}^{2} \theta\right)$. The modulation disappears, simulating a square detector and removing the nonactive belts along the $y$ axis where the columns are. The modulation phase can be shifted from the expected $90^{\circ}$ if the efficiency of the RPCs is not uniform on the entire carpet.

\section{B. Magnetic effect}

The magnetic effect was studied by means of $12 \mathrm{CR}$ beams with the same values of primary energy $(3 \mathrm{TeV})$, zenith angle $\left(45^{\circ}\right)$, and interaction height $(19 \mathrm{~km})$. The choice of the zenith angle allows coverage of the full $\sin \xi$ range. The energy has been increased with respect to the previous simulation in order to compensate the larger path in the atmosphere. Different azimuth angles (see Table I) have been used in order to get different values of the $\xi$ angle. Three intensities of the magnetic field have been used: 0.0, 49.7 (the actual GeoMF at the YBJ site), and $99.4 \mu \mathrm{T}$ (twice the actual GeoMF).

The shower cores have been reconstructed by using separate negative and positive particles. The results validate Eq. (5) because the distance between the positive and negative cores increases linearly with $\sin \xi$ and $B$ (Fig. 3, left). As expected, on the basis of the simple model, when

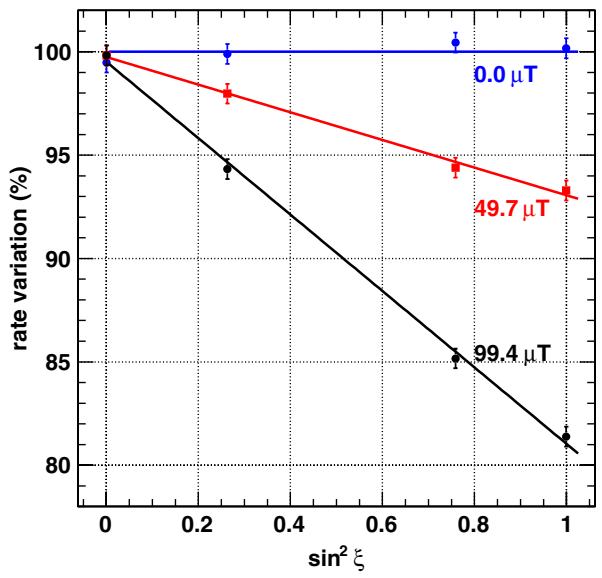

FIG. 3 (color online). Simulation. Left: mean values of the positive-negative core distance versus sin $\xi$ for different magnetic fields. A linear fit is superimposed. Right: fractional rate variation versus $\sin ^{2} \xi$ for different magnetic fields. A linear fit is superimposed. 
the shower axis is nearly on the bending plane $\left(\theta=45^{\circ}\right.$, $\phi=251.5^{\circ}, v_{\|}=0$ ), the positive and negative cores are shifted truly on the WE axis with a typical shift of the order of $4.5 \mathrm{~m}$ for each particle.

It has been also verified that the effect of the shower stretching on the reconstruction of the EAS direction is negligible, whereas it is significant on the trigger efficiency. Neglecting the detector effect, the rate is dependent on $\sin ^{2} \xi$. The right plot in Fig. 3 can be fitted according to the following function:

$$
\lambda=\lambda_{\max }\left(1-\eta \sin ^{2} \xi\right) .
$$

It results also that the term $\eta$ depends linearly on $B^{2}$. Thus, we conclude that the rate reduction is proportional to $B^{2} \sin ^{2} \xi$. Therefore, the rate decrease is related to the GeoMF stretching of the EAS footprint. In other words, the decrease of the charge density close to the core actually reduces the trigger efficiency when the core is on the array.

To explain this result, we must take into account that the enlargement of the lateral distribution has two contrary effects: the more separated particles can fire more pads; on the contrary, the enlargement can reduce the number of hits on the central carpet (trigger area). Looking at the simulated events, we verify that the second one is the leading effect; therefore, the trigger reduction is essentially a boundary effect.

Before closing the section, we want remind that the CRbeam simulations do not allow a full estimate of the GeoMF and detector effects but indicate only their functional shape. The amplitudes of these effects are precisely determined by the analysis of the real data sample.

\section{WHAT TO EXPECT}

Combining Eqs. (6) and (7), the number of showers $\left(N_{\theta}\right)$ with cores on carpet in an angular window $\Delta \theta \times \Delta \phi$ around a fixed zenith angle is

$$
N_{\theta}=N_{\theta, \max }\left(1-\eta \sin ^{2} \xi\right)\left\{1+g_{2 A} \cos \left[2\left(\phi-\phi_{2 A}\right)\right]\right\},
$$

where $N_{\theta, \max }$ is the number of events expected without a magnetic field (the average value with respect to $\phi$ ).

At the beginning, we can neglect the detector factor in order to point out the GeoMF effect. Equation (8) becomes

$$
N_{\theta}=N_{\theta, \max }\left(1-\eta \sin ^{2} \xi\right)
$$

where the angle $\xi$ can be calculated by using the direction cosines of the shower $(l, m$, and $n)$ and those of the GeoMF $\left(l_{B}, m_{B}\right.$, and $\left.n_{B}\right)$. The formalism can be simplified by introducing another azimuth angle $\Phi$ defined as $\Phi=\phi-\phi_{B}$. According to this definition, $\Phi_{B}=0$; therefore, the angle $\xi$ is given by the equation

$$
\begin{aligned}
\cos \xi & =l l_{B}+m m_{B}+n n_{B}= \\
& =\sin \theta \sin \theta_{B} \cos \Phi+\cos \theta \cos \theta_{B} .
\end{aligned}
$$

Then, it is straightforward to calculate

$$
\sin \xi=\sqrt{A_{0}+A_{1} \cos \Phi+A_{2} \cos (2 \Phi)},
$$

where

$$
\begin{aligned}
& A_{0}=\sin ^{2} \theta_{B}+\left(1-\frac{3}{2} \sin ^{2} \theta_{B}\right) \sin ^{2} \theta \\
& A_{1}=-\frac{1}{2} \sin 2 \theta_{B} \sin 2 \theta \\
& A_{2}=-\frac{1}{2} \sin ^{2} \theta_{B} \sin ^{2} \theta
\end{aligned}
$$

The first term is positive, whereas $A_{1}$ and $A_{2}$ are negative. A two-harmonics function is obtained combining Eqs. (9) and (10) and referring back to the previous azimuth angle

$$
N_{\theta}=N_{\theta, 0}\left\{1+g_{1} \cos \left(\phi-\phi_{1}\right)+g_{2} \cos \left[2\left(\phi-\phi_{2}\right)\right]\right\} \text {, }
$$

where

$$
\begin{gathered}
\phi_{1}=\phi_{2}=\phi_{B}, \\
N_{\theta, 0}=N_{\theta, \text { max }}\left(1-\eta A_{0}\right), \\
g_{1}=-\frac{\eta A_{1}}{1-\eta A_{0}}=\frac{\eta \sin 2 \theta_{B}}{2\left(1-\eta A_{0}\right)} \sin 2 \theta, \\
g_{2}=-\frac{\eta A_{2}}{1-\eta A_{0}}=\frac{\eta \sin ^{2} \theta_{B}}{2\left(1-\eta A_{0}\right)} \sin ^{2} \theta .
\end{gathered}
$$

$N_{\theta, 0}$ in Eq. (14) represents the $\phi$ average at fixed $\theta$ of the number of events in $\phi$ bins reduced by the effect of shower stretching on the trigger, and $\eta A_{0}$ is the reduction percentage.

Taking into account also the detector factor according to Eq. (8) and assuming $g_{1} g_{2 A} \simeq 0, g_{2} g_{2 A} \simeq 0$, Eq. (12) becomes

$$
\begin{aligned}
N_{\theta}= & N_{\theta, 0}\left\{1+g_{1} \cos \left(\phi-\phi_{1}\right)+g_{2} \cos \left[2\left(\phi-\phi_{2}\right)\right]\right. \\
& \left.+g_{2 A} \cos \left[2\left(\phi-\phi_{2 A}\right)\right]\right\},
\end{aligned}
$$

where the first harmonic is exclusively due to the GeoMF, and the second harmonic is split in two terms: one with amplitude $g_{2}$ and phase $\phi_{2}$ is due to the GeoMF; the other one with amplitude $g_{2 A}$ and phase $\phi_{2 A}$ is due to the detector acceptance. 


\section{ANALYSIS OF INTERNAL-CORE SHOWERS}

\section{A. Data sample}

The data set was collected during the period October $7-14,2010\left(6.77 \times 10^{5} \mathrm{~s}\right)$. The RPC efficiency was monitored by means of a five-layer telescope [23], and in that period it was $\sim 96.7 \%$.

Two analysis cuts were applied in order to select internalcore events: the shower core must be reconstructed inside a square of $40 \times 40 \mathrm{~m}^{2}$ at the center of the carpet, and the zenith angle must be lower than $60^{\circ}$. The first cut was chosen in order to make more evident the trigger efficiency decrease (the effect is expected very different for showers with cores far from the detector). The second one simplifies the analysis because in that zenith angle range, the detector effect does not prevail on the magnetic one. These cuts guarantee also a more reliable reconstruction of the shower direction. After these cuts, more than $347 \times 10^{6}$ events survive with a mean rate of $512.8 \mathrm{~Hz}$. A very short analysis has been performed also for external-core showers (Sec. VII).

\section{B. Timing calibration}

Small errors in the reconstruction of the shower direction may introduce large systematic errors in the azimuthal distribution, especially for small zenith angles. Thus, the 18,360 pads were carefully time calibrated by means of a software procedure based on the characteristic plane method [24].

\section{Rate versus $\sin ^{2} \xi$}

The dependence of the number of events on $\sin ^{2} \xi$ is well represented in Fig. 4, where the number of events in an angular window $\Delta \theta \times \Delta \phi=2^{\circ} \times 5^{\circ}$ is plotted versus $\sin ^{2} \xi$. The $\theta$ value is fixed for each aligned group of points while $\phi$ is running. The $\sin ^{2} \xi$ range depends on $\theta$ (see Fig. 1) being maximum for $\theta=45^{\circ}$ and minimum for $\theta$ close to $0^{\circ}$. The scattered points can be fitted by means of function (9), which is then confirmed to give a good description of the data. This fit does not separate GeoMF and detector effects, and we have analytically verified that $\eta$ would be overestimated. Therefore, a fit has been implemented by using function (8) with four parameters $\left(N_{\theta, \max }\right.$, $\eta, g_{2 A}$, and $\left.\phi_{2 A}\right)$. For the lower values of the zenith angle, the fit is not reliable because the detector effect is too small. Then we used a two-step fit: from the fit for the zenith angles higher than $12^{\circ}$, we estimate the phase $\phi_{2 A}=96.5^{\circ} \pm 3.4^{\circ}$ (Fig. 5). The fit errors are large, but the parameter value is very stable. Then we fix $\phi_{2 A}$ in function (8) and perform the fit in the whole $\theta$ range. The fit results for $\eta$ and $g_{2 A}$ are shown in Fig. 6 ; the first point $\left(\theta \sim 1^{\circ}\right)$ is not displayed because the $\sin ^{2} \xi$ range is too small, the uncertainty on $\phi$ is very high, and then the fit is not reliable for this zenith value.

As a result of this fit, we conclude that $\eta=(4.093 \pm$ $0.068) \%$ does not show any dependence on the zenith

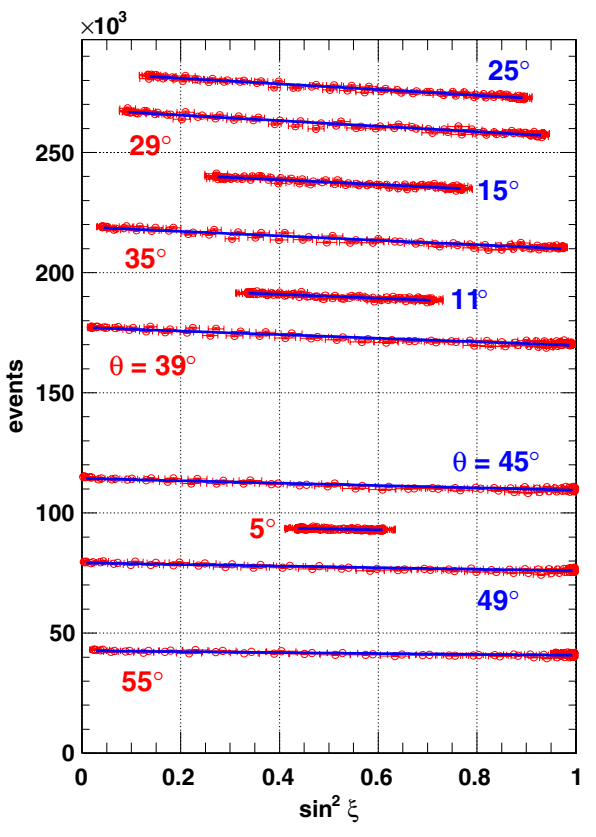

FIG. 4 (color online). Real data: scatter plot of the number of events in angular windows $\Delta \theta \times \Delta \phi=2^{\circ} \times 5^{\circ}$ versus $\sin ^{2} \xi$ for different values of $\theta$. Fits with function (9) are superimposed.

angle, and the detector coefficient $g_{2 A}$ has the expected dependence on the zenith angle $\left(g_{2 A}=\operatorname{ttg}^{2} \theta\right)$.

By using Eq. (8) in order to confirm the validity of this procedure, we have defined a corrected number of events

$$
\begin{aligned}
N_{\theta}^{*} & \equiv N_{\theta} /\left\{1+g_{2 A} \cos \left[2\left(\phi-\phi_{2 A}\right)\right]\right\} \\
& =N_{\theta, \max }\left(1-\eta \sin ^{2} \xi\right),
\end{aligned}
$$

where the detector parameters $\left(g_{2 A}, \phi_{2 A}\right)$ of the previous fit have been used to calculate $N_{\theta}^{*}$. As an example, the scatter plots of $N_{\theta}$ and $N_{\theta}^{*}$ versus $\sin ^{2} \xi$ are shown in Fig. 7 for

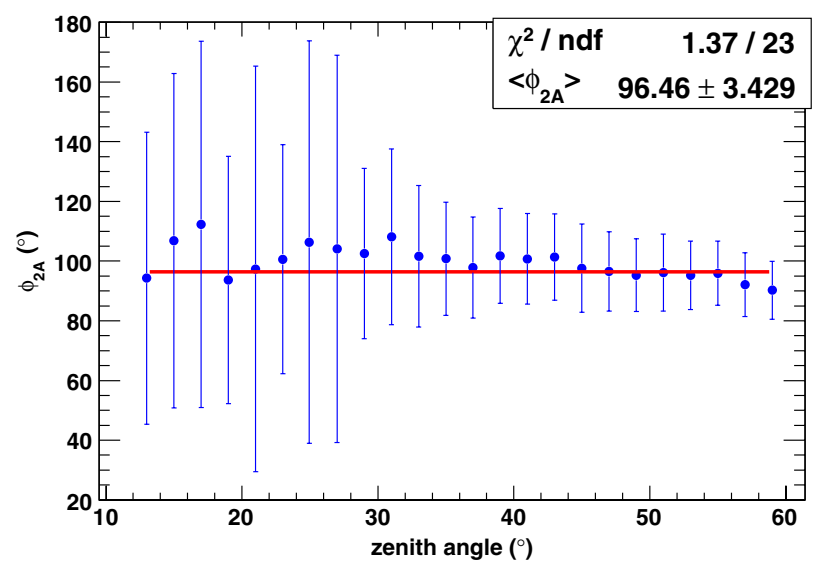

FIG. 5 (color online). Real data: the phase $\left(\phi_{2 A}\right)$ of the detector effect versus the zenith angle. The values of $\phi_{2 A}$ are estimated by means of a fit of $N_{\theta}$ versus $\sin ^{2} \xi$ and $\phi$ using function (8). Below $30^{\circ}$ in the zenith, the phase shows large errors due to the vanishing small size of the coefficient $g_{2 A}$ (see Fig. 6, right). 

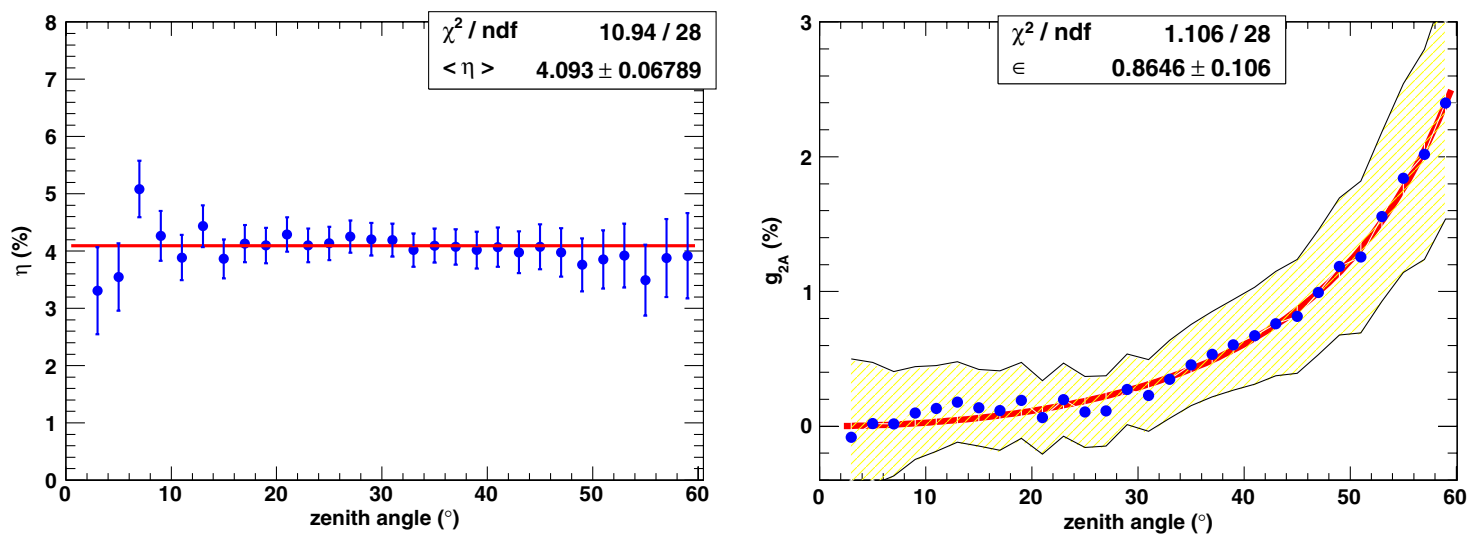

FIG. 6 (color online). Real data: the coefficients $\eta$ (left) and $g_{2 A}$ (right) versus $\theta$. The values of $\eta$ and $g_{2 A}$ are estimated by fitting $N_{\theta}$ versus $\sin ^{2} \xi$ and $\phi$ according to function (8). In the right plot, the colored area represents $1 \sigma$ uncertainty, and the fit according to $g_{2 A}=\operatorname{tg}^{2} \theta$ is superimposed.

$\theta=35^{\circ}$. The fit with functions (9) and (18) works better for $N_{\theta}^{*}\left(\chi^{2} / n d f=1.2\right)$ than for $N_{\theta}\left(\chi^{2} / n d f=3.2\right)$. This result confirms that the detector effect has been properly estimated. Similar improvements are observed for the other $\theta$ values.

\section{Azimuthal distribution}

The azimuthal distribution obtained by integrating all the showers in the range $\theta<60^{\circ}$ can be fitted by the double harmonic function (12). The fit result is shown in Fig. 8; the phase of the first harmonic $\left(\phi_{1}=72.75^{\circ} \pm 0.29^{\circ}\right)$ is compatible within $3 \sigma$ with the GeoMF azimuth $\left(\phi_{B}=71.89^{\circ}\right)$, as expected if the origin of the modulation is geomagnetic. This is not the case of the second-harmonic phase $\left(\phi_{2}=87.00^{\circ} \pm 0.40^{\circ}\right)$ with a value in between those expected for the detector and GeoMF effects.

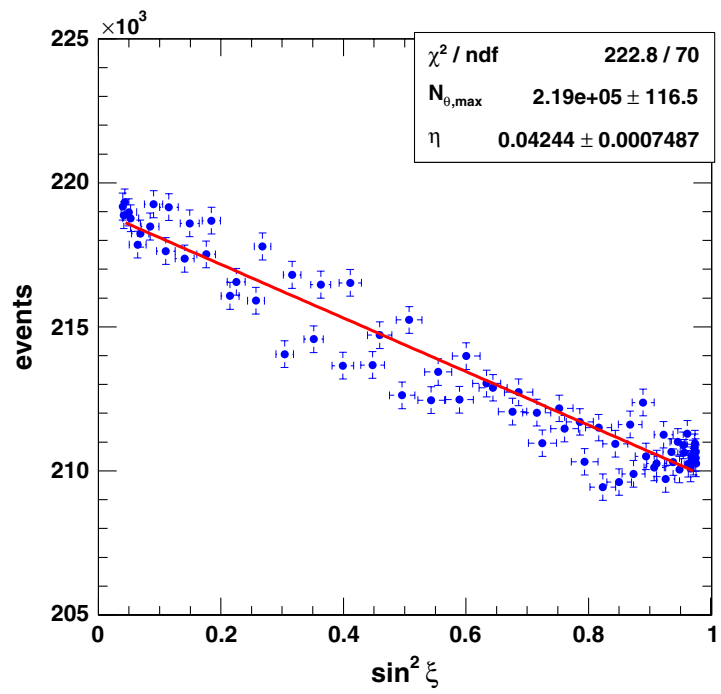

The high value of $\chi^{2} / n d f$ is mainly due to some inefficiencies at $\phi \sim n 90^{\circ}(n=0,1,2,3)$. The origin of these dips is not fully clear. Their position in the azimuthal distribution indicates a relation with the detector shape and the framework of the experimental hall. We suppose they could be shadows of iron beams and columns. Anyway, the presence of these dips does not invalidate this analysis on the GeoMF effect. To take into account the dips, we assume that they have a Gaussian shape (with angular width $\sigma$ and amplitude $k$ ). Then the fit is performed with the following function:

$$
\begin{aligned}
N= & N_{0}\left\{1+g_{1} \cos \left(\phi-\phi_{1}\right)+g_{2} \cos \left[2\left(\phi-\phi_{2}\right)\right]\right. \\
& +k\left(e^{-\phi^{2} / 2 \sigma^{2}}+e^{-(\phi-90)^{2} / 2 \sigma^{2}}+e^{-(\phi-180)^{2} / 2 \sigma^{2}}\right. \\
& \left.\left.+e^{-(\phi-270)^{2} / 2 \sigma^{2}}+e^{-(\phi-360)^{2} / 2 \sigma^{2}}\right)\right\} .
\end{aligned}
$$

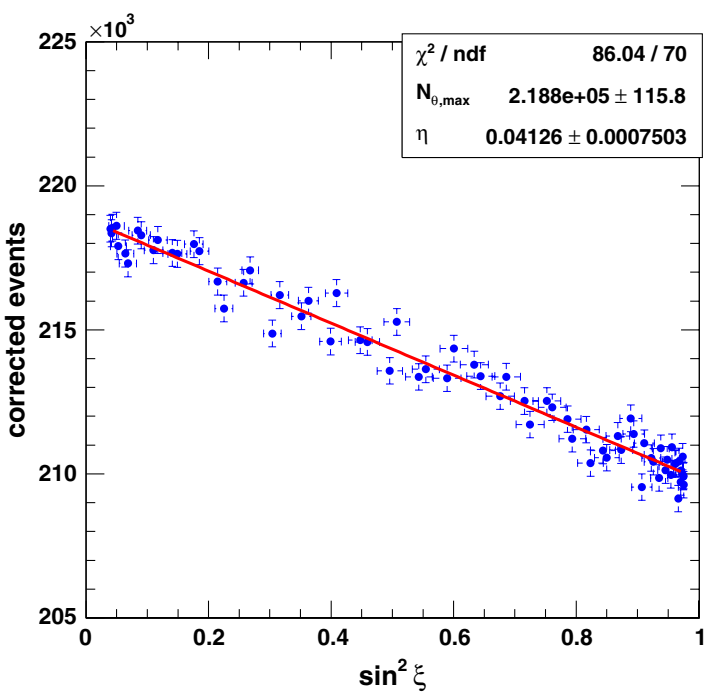

FIG. 7 (color online). Real data in angular windows $\Delta \theta \times \Delta \phi=2^{\circ} \times 5^{\circ}$ centered at $\theta=35^{\circ}$. Left: scatter plot of the number of events $\left(N_{\theta}\right)$ versus $\sin ^{2} \xi$ fitted with function (9). Right: scatter plot of the corrected number of events $\left(N_{\theta}^{*}\right)$ versus $\sin ^{2} \xi$, the correction and fit are performed according to function (18). A sharp reduction of the $\chi^{2} / n d f$ value is visible when the corrected number of events is used. 

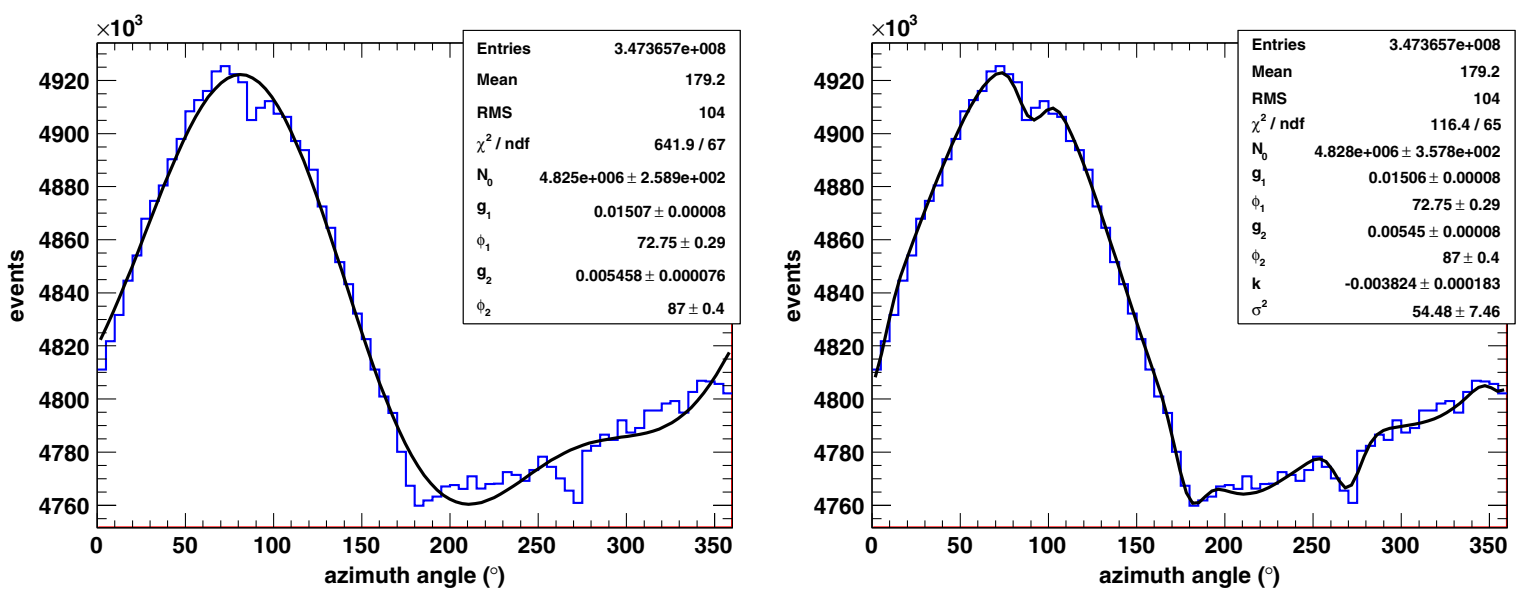

FIG. 8 (color online). Real data: the azimuthal distribution fitted with function (12) (left) and function (19) (right).

The result of the new fit is shown in the right panel of Fig. 8. The $\chi^{2} / n d f$ becomes smaller $(116 / 65=1.8)$ with respect to that $(642 / 67=9.6)$ of the fit in the left panel of Fig. 8. The parameters $g_{1}, \phi_{1}, g_{2}$, and $\phi_{2}$ do not change in this second fit. The dip amplitude is $k=-(0.382 \pm 0.018) \%$, and the Gaussian width is $\sigma=7.38^{\circ} \pm 0.51^{\circ}$.

\section{E. Amplitudes $g_{1}$ and $g_{2}$ as functions of $\theta$}

The azimuthal distribution has been studied also in $\theta$ ranges of $2^{\circ}$ in order to check the dependence of $g_{1}$ and $g_{2}$ on $\theta$. Also, in this case, function (12) has been used. The result for $g_{1}$ is shown in Fig. 9. By fitting the experimental points with function (15), we can confirm that $\eta$ does not depend on $\theta$, and its estimate is very close to the previous one (Fig. 6, left). The fractional variation of the term $\left(1-\eta A_{0}\right)$ is less than $0.7 \%$. Then, $g_{1}$ is mainly proportional to $\sin 2 \theta$.

According to Eq. (16), $g_{2}$ is expected to be mainly proportional to $\sin ^{2} \theta$; however, the data do not match with the expected shape, and the value of $\eta$ from the fit is in

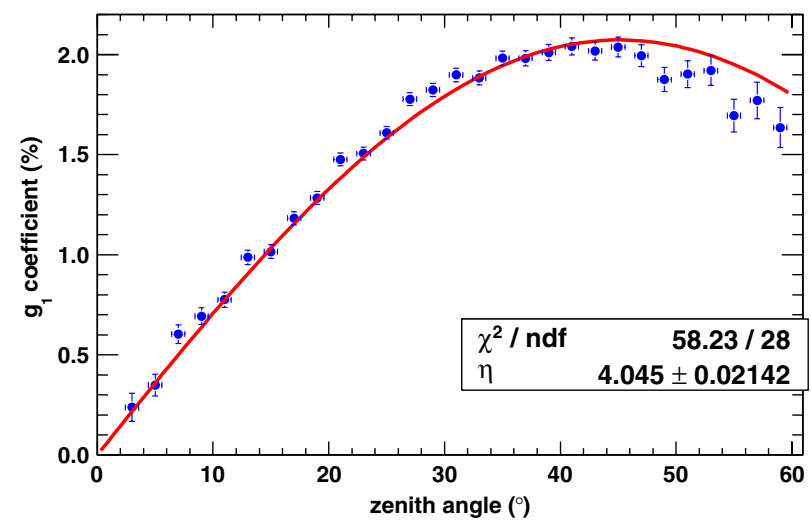

FIG. 9 (color online). Real data: the coefficient $g_{1}$ versus the zenith angle. The fit with function (15) has been applied. disagreement with the previous estimates. This result is not odd because we know that the GeoMF and detector effects are not disentangled in the second harmonic of function (12).

\section{F. Two components in the second harmonic}

While the first harmonic is in full agreement with the GeoMF model, this is not the case for the second harmonic. The tension can be solved by simply taking into account that the detector effect operates on the second harmonic and that the proper function to fit the azimuthal distribution is function (17). Thus, three different data sets were selected on the basis of the zenith angle in order to disentangle the two second-harmonic components. The $\phi$ distributions of the subsamples (named $\alpha$ for $\theta<20^{\circ}, \beta$ for $20^{\circ}<\theta<40^{\circ}$, and $\gamma$ for $40^{\circ}<\theta<60^{\circ}$ ) are shown in Fig. 10 and can be fitted all together with a single function:

$$
\begin{aligned}
N_{i}= & N_{i, 0}\left\{1+\frac{\eta \sin 2 \theta_{B}}{2\left(1-\eta A_{0}\right)}\langle\sin 2 \theta\rangle_{i} \cos \left(\phi-\phi_{1}\right)\right. \\
& +\frac{\eta \sin ^{2} \theta_{B}}{2\left(1-\eta A_{0}\right)}\left\langle\sin ^{2} \theta\right\rangle_{i} \cos \left[2\left(\phi-\phi_{1}\right)\right] \\
& \left.+g_{2 A}^{i} \cos \left[2\left(\phi-\phi_{2 A}\right)\right]\right\},
\end{aligned}
$$

where the coefficients of the magnetic components $\left(g_{1}\right.$ and $\left.g_{2}\right)$ are deduced from Eqs. (15) and (16), the phase $\phi_{1}$ is used for the first and second magnetic harmonics, and the index $i=\alpha, \beta, \gamma$ indicates the subsamples. Then, the fit parameters are $N_{\alpha, 0}, N_{\beta, 0}, N_{\gamma, 0}, \eta$, $\phi_{1}, g_{2 A}^{\alpha}, g_{2 A}^{\beta}, g_{2 A}^{\gamma}$, and $\phi_{2 A}$. The new fit works very well (the results are shown in Table II); however, the $\chi^{2} / n d f$ value is higher $(1053 / 207=5.1)$ when the dip correction is not applied. It reduces to $481 / 201=2.4$ by adding the negative Gaussians for the dips. The phase $\phi_{1}$ and the GeoMF azimuth $\phi_{B}$ are in agreement; 

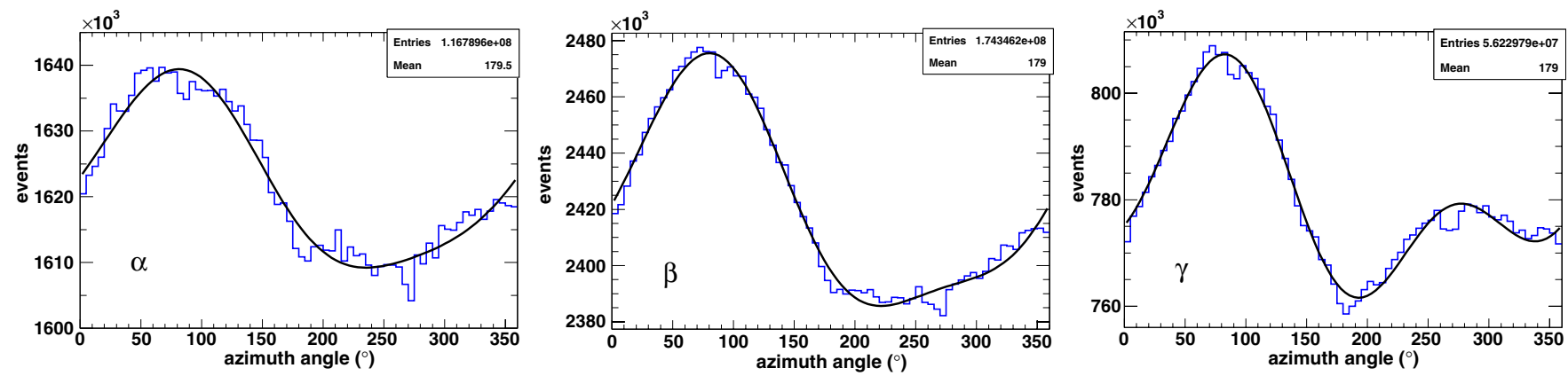

FIG. 10 (color online). Real data: the azimuthal distribution in different $\theta$ ranges $\left(0^{\circ}-20^{\circ}, 20^{\circ}-40^{\circ}\right.$, and $\left.40^{\circ}-60^{\circ}\right)$. The fit with function (20) is superimposed (see Table II for the fit parameters).

the $\eta$ value is fully compatible with the previous estimates. The coefficients $g_{2 A}^{i}$ increase with $\theta$ and $\phi_{2 A} \simeq 96^{\circ}$ according to the results of the previous fit (Figs. 5 and 6 , right).

\section{G. Final remarks}

The azimuthal distribution depends on a mix of magnetic and detector effects, whose contributions are shown in Fig. 11 where the coefficients $g_{1}, g_{2}$, and $g_{2 A}$ are plotted as functions of the zenith angle according to formulas (15) and (16), Fig. 6, right, and Table II. This plot suggests that the GeoMF origin of the rate reduction is leading with respect to the detector effect in the zenith range $20^{\circ}-40^{\circ}$, where $g_{2 A} \ll g_{1}$. For $\theta>45^{\circ}$, the increase of $g_{2}$ and $g_{2 A}$ and the decrease of $g_{1}$ suggest that the second harmonic is dominant in the azimuthal distribution at high zenith angles. We verified this in the data by fitting the azimuthal distribution in the range $60^{\circ}<\theta<80^{\circ}$. The amplitude of the second-harmonic is almost 4 times greater than the amplitude of the first one.

The measurement of $\eta$ has been repeated with three different methods obtaining compatible results. We conclude that $\eta=(4.055 \pm 0.014) \%$ is the proper estimate for this analysis of the ARGO-YBJ data. Different values are expected applying other cuts on the core position (see the next section). The coefficient $\eta$ depends also on the geomagnetic latitude and altitude of the site, detector features, and trigger requirements.

TABLE II. Results of the fit with function (20) of the distributions in Fig. 10.

\begin{tabular}{ll}
\hline \hline$\eta(\%)$ & $4.060 \pm 0.019$ \\
$\phi_{1}\left({ }^{\circ}\right)$ & $72.22 \pm 0.28$ \\
$g_{2 A}^{\alpha}(\%)$ & $0.124 \pm 0.013$ \\
$g_{2 A}^{\beta}(\%)$ & $0.271 \pm 0.011$ \\
$g_{2 A}^{\gamma}(\%)$ & $1.076 \pm 0.019$ \\
$\phi_{2 A}\left({ }^{\circ}\right)$ & $96.30 \pm 0.47$ \\
\hline \hline
\end{tabular}

\section{EXTERNAL-CORE SHOWERS}

An increase of the charge density is expected far from the EAS core, as a counterbalance to the decrease close to the cores (see, also, Ref. [3]). Then, a GeoMF increase of the trigger efficiency is expected for showers with core outside the ARGO-YBJ carpet. In analogy with Eq. (9), the number of external events $\left(N_{\theta}^{\text {out }}\right)$ in an angular window $\Delta \theta \times \Delta \phi$ around a fixed zenith angle may depend on $\xi$ as

$$
N_{\theta}^{\text {out }}=N_{\theta, \min }^{\text {out }}\left(1+\eta_{\text {out }} \sin ^{2} \xi\right) \text {, }
$$

where $N_{\theta, \text { min }}^{\text {out }}$ is the number of events expected without a magnetic field, and $\eta_{\text {out }}$ is the parameter analogous to $\eta$ (with opposite sign).

A sample of external showers was selected requiring a core reconstructed outside a $120 \mathrm{~m}$ square centered on the ARGO-YBJ carpet. A short analysis confirms the validity of Eq. (21). Indeed, Fig. 12 is very similar to Fig. 4 with opposite slopes. The value of $\eta_{\text {out }}$ is $\sim 2.5 \%$.

\section{ABSOLUTE POINTING ACCURACY OF AN EAS ARRAY}

The Moon shadow analysis [25] is commonly used to check the absolute pointing of EAS arrays. It requires

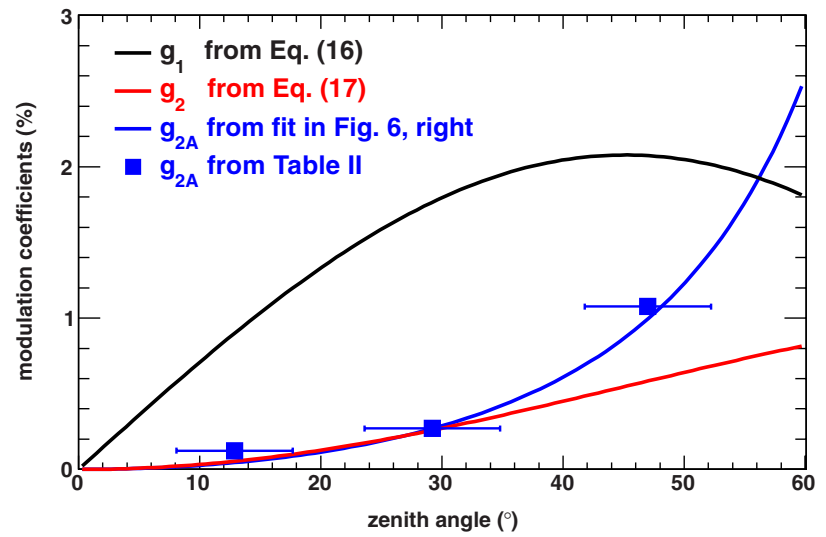

FIG. 11 (color online). Real data: the coefficients $g_{1}, g_{2}$, and $g_{2 A}$ versus $\theta$ from formulas (15) and (16), Table II, and the fit in Fig. 6, right. The experimental points are drawn at the mean $\theta$ value and the error bars are the RMS of the $\theta$ distribution. 


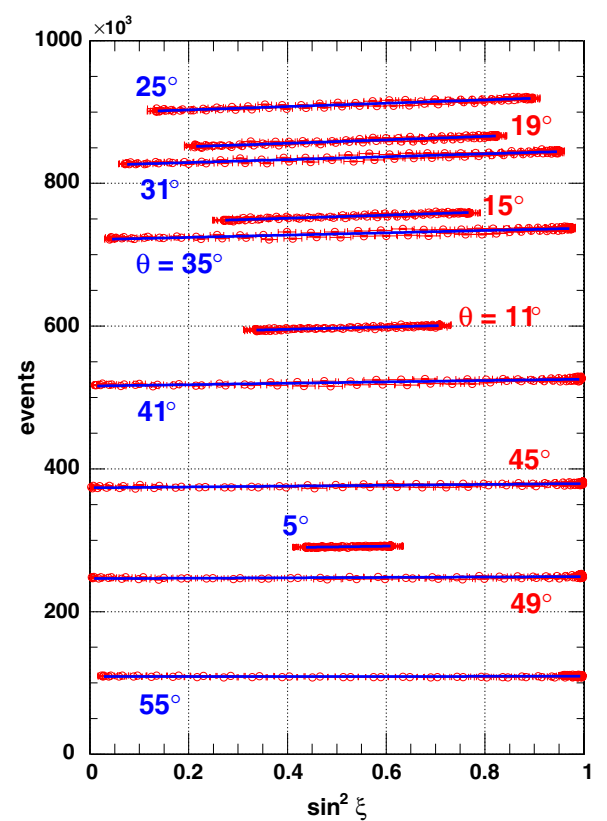

FIG. 12 (color online). Showers with cores outside the detector area: scatter plot of the number of events in angular windows $\Delta \theta \times \Delta \phi=2^{\circ} \times 5^{\circ}$ versus $\sin ^{2} \xi$ for different values of $\theta$. Fits with function (21) are superimposed.

very large samples of showers, and the pointing accuracy cannot be checked daily. The GeoMF effect on EAS suggests another method to control the array pointing accuracy. Indeed, only the first harmonic of the azimuthal distribution contributes to the mean values of the direction cosines $(\langle l\rangle,\langle m\rangle)$, and a fast check of the $\phi_{1}$ value is possible also using small samples of internalcore events:

$$
\phi_{1}=\arctan \frac{\langle m\rangle}{\langle l\rangle} .
$$

When $\phi_{1} \neq \phi_{B}$, a systematic correction is necessary. The direction cosines must be increased or reduced in order to get the expected mean values

$$
\begin{array}{r}
\langle l\rangle=\frac{\eta \sin 2 \theta_{B}}{4} \cos \phi_{B}\left\langle\frac{\sin 2 \theta \sin \theta}{1-\eta A_{0}}\right\rangle, \\
\langle m\rangle=\frac{\eta \sin 2 \theta_{B}}{4} \sin \phi_{B}\left\langle\frac{\sin 2 \theta \sin \theta}{1-\eta A_{0}}\right\rangle .
\end{array}
$$

The only requirement to perform the pointing correction is to estimate precisely the reduction factor $\eta$ for the data sample used in the analysis.

\section{CONCLUSIONS}

The effect of the geomagnetic Lorentz force on EAS charged particles has been studied in a data sample collected by the ARGO-YBJ experiment. The shower extension enlarges depending on the arrival direction with respect to the GeoMF and the modified density of charged particles slightly reduces the trigger efficiency for EAS with cores on the detector. The GeoMF effect and the trigger efficiency decrease are understood by means of a model complemented by Monte Carlo simulations and verified in a large real data sample.

The nonuniform azimuthal distribution was deeply studied. It is well described by two harmonics: the first one of about $1.5 \%$ and the second one of about $0.5 \%$. The first harmonic is due to the GeoMF; the second one is the sum of the magnetic and detector effects. The measurement of the geomagnetic phase $\left(\phi_{1}=72.22^{\circ} \pm 0.28^{\circ}\right)$ is fully compatible with the expected value $\left(\phi_{B}=71.89^{\circ}\right)$. Other checks confirm the geomagnetic origin of the modulation. A GeoMF effect with opposite sign has been observed also for showers with cores outside the detector area.

The phase of the first harmonic $\left(\phi_{1}\right)$ can be used as a marker of the absolute pointing of EAS arrays. When necessary, a simple correction of the absolute pointing can be applied.

To our knowledge, the experimental results presented here are the first evidence of the charge density reduction near the EAS axis due to the GeoMF.

\section{ACKNOWLEDGMENTS}

This work is supported in China by the NSFC (Grants No. 10120130794 and No. 11205165), the Chinese Ministry of Science and Technology, the Chinese Academy of Sciences, the Key Laboratory of Particle Astrophysics, CAS, and in Italy by the Istituto Nazionale di Fisica Nucleare (INFN). We also acknowledge the essential support of W. Y. Chen, G. Yang, X. F. Yuan, C. Y. Zhao, R. Assiro, B. Biondo, S. Bricola, F. Budano, A. Corvaglia, B. D’Aquino, R. Esposito, A. Innocente, A. Mangano, E. Pastori, C. Pinto, E. Reali, F. Taurino, and A. Zerbini in the installation, debugging, and maintenance of the detector. 
[1] T. H. Johnson, Phys. Rev. 43, 834 (1933); L. W. Alvarez and A. H. Compton, Phys. Rev. 43, 835 (1933); T. H. Johnson and E. C. Stevenson, Phys. Rev. 44, 125 (1933).

[2] G. Cocconi, Phys. Rev. 93, 646 (1954); 95, 1705(E) (1954).

[3] A. A. Ivanov, V. P. Egorova, V. A. Kolosov, A. D. Krasil'nikov, M. I. Pravdin, and I. E. Sleptsov, JETP Lett. 69, 288 (1999).

[4] M. Bahmanabadi, A. Anvari, M. Khakian Ghomi, D. Purmohammad, J. Samimi, and M. L. Rachti, Exp. Astron. 13, 39 (2002); M. Khakian Ghomi, M. Bahmanabadi, J. Samimi, A. Anvari, and F. Sheidaei, in Proceedings of the 30th International Cosmic Ray Conference, Merida, 2007 edited by R. Caballero, J. C. D'Olivo, G. Medina-Tanco, L. Nellen, F. A. Sánchez, and J. F. Valdés-Galicia, (Universidad Nacional Autónoma de México, Mexico, 2008) Vol. 4, p. 11.

[5] D. Ardouin et al. (CODALEMA Collaboration), Astropart. Phys. 31, 192 (2009).

[6] A. Cillis and S. J. Sciutto, J. Phys. G 26, 309 (2000).

[7] S. C. Commichau, A. Biland, J. L. Contreras, R. de los Reyes, A. Moralejo, J. Sitarek, and D. Sobczyńska, Nucl. Instrum. Methods Phys. Res., Sect. A 595, 572 (2008).

[8] J. N. Capdevielle, C. Le Gall, Kh. N. Sanosyan, Astropart. Phys. 13, 259 (2000).

[9] P. Abreu et al. (Pierre Auger Collaboration), J. Cosmol. Astropart. Phys. 11 (2011) 022.

[10] H. H. He et al., in Proceedings of the 29th International Cosmic Ray Conference, Pune, 2005 Vol. 6, pp. 5-8.

[11] P. Bernardini et al., arXiv:1110.0670; P. Bernardini and S. N. Sbano, J. Phys. Conf. Ser. 409, 012229 (2013); S. N. Sbano, Nucl. Phys. B, Proc. Suppl. 239-240, 274 (2013).

[12] G. Aielli et al. (ARGO-YBJ Collaboration), Nucl. Instrum. Methods Phys. Res., Sect. A 661, S50 (2012).

[13] G. Aielli et al. (ARGO-YBJ Collaboration), Astrophys. J. Lett. 714, L208 (2010); B. Bartoli et al. (ARGO-YBJ Collaboration), Astrophys. J. 734, 110 (2011); B. Bartoli et al. (ARGO-YBJ Collaboration), Astrophys. J. Lett. 745, L22 (2012); B. Bartoli et al. (ARGO-YBJ Collaboration), Astrophys. J. 758, 2 (2012); B. Bartoli et al. (ARGO-YBJ Collaboration), Astrophys. J. 760, 110 (2012); B. Bartoli et al. (ARGO-YBJ Collaboration), Astrophys. J. 767, 99 (2013); B. Bartoli et al. (ARGO-YBJ Collaboration), Astrophys. J. 779, 27 (2013).
[14] G. Aielli et al. (ARGO-YBJ Collaboration), Phys. Rev. D 80, 092004 (2009); G. Aielli et al. (ARGO-YBJ Collaboration), Astrophys. J. 729, 113 (2011); B. Bartoli et al. (ARGO-YBJ Collaboration), Phys. Rev. D 85, 022002 (2012); B. Bartoli et al. (ARGO-YBJ Collaboration), Phys. Rev. D 85, 092005 (2012); B. Bartoli et al. (ARGO-YBJ Collaboration), Phys. Rev. D 88, 082001 (2013).

[15] G. Aielli et al. (ARGO-YBJ Collaboration), Nucl. Instrum. Methods Phys. Res., Sect. A 562, 92 (2006).

[16] M. Iacovacci et al. (ARGO-YBJ Collaboration), in Proceedings of the 33rd International Cosmic Ray Conference, Rio de Janeiro, 2013 (Report No. CR-IN 1035).

[17] G. Aielli et al. (ARGO-YBJ Collaboration), Astropart. Phys. 30, 85 (2008); G. Aielli et al. (ARGO-YBJ Collaboration), Astrophys. J. 699, 1281 (2009).

[18] www.ngdc.noaa.gov.

[19] D. Heck et al., Report No. FZKA 6019, 1998 [see, also, http://www-ik.fzk.de/ corsika/physics_description/ corsika_phys.html].

[20] R. Engel, T. K. Gaisser, T. Stanev, and P. Lipari, in Proceedings of the 26th International Cosmic Ray Conference, Salt Lake City, 1999 edited by D. B. Kieda, M. B. Salamon, and B. L. Dingus, (AIP Conference Proceedings, New York, 1999) Vol. 1, p. 415; E. J. Ahn, R. Engel, T. K. Gaisser, P. Lipari, and T. Stanev, Phys. Rev. D 80, 094003 (2009).

[21] A. Fassò, P. R. Sala, A. Fasso, J. Ranft, Reports No. CERN2005-10, 2005, No. INFN/TC_05/11, and No. SLAC-R773, www.slac.stanford.edu/cgi-wrap/getdoc/slac-r-773.pdf; G. Battistoni, F. Cerutti, A. Fassò, A. Ferrari, S. Muraro, J. Ranft, S. Roesler, and P. R. Sala, AIP Conf. Proc. 896, 31 (2007); G. Battistoni, A. Margiotta, S. Muraro, and M. Sioli, Nucl. Instrum. Methods Phys. Res., Sect. A 626-627, S191 (2011) [see, also, www.fluka.org].

[22] CERN Report No. W5013, 1993.

[23] G. Aielli et al. (ARGO-YBJ Collaboration), Nucl. Instrum. Methods Phys. Res., Sect. A 608, 246 (2009).

[24] H. H. He, P. Bernardini, A. K. Calabrese Melcarne, and S. Z. Chen, Astropart. Phys. 27, 528 (2007); G. Aielli et al. (ARGO-YBJ Collaboration), Astropart. Phys. 30, 287 (2009).

[25] B. Bartoli et al. (ARGO-YBJ Collaboration), Phys. Rev. D 84, 022003 (2011). 